\title{
Procesamiento gráfico de datos obtenidos en la simulación de redes. Análisis comparativo de herramientas como complemento del simulador
} NS-2

\author{
Graphical processing of data derived from network simulation \\ Comparative analysis of tools as a complement to the NS-2 simulator
}

\begin{abstract}
María Luz Almada, Carlos Alberto Talay
mluzalmada@gmail.com,ctalay@uarg.unpa.edu.ar

Instituto de Tecnología Aplicada - Unidad Académica Río Gallegos - Universidad Nacional de la Patagonia Austral - Av. Gregores y Piloto "Lero" Rivera - Río Gallegos - Santa Cruz -

Argentina
\end{abstract}

Recibido: 15/02/2019. Aceptado: 22/08/2019

\section{RESUMEN}

El procesamiento de los datos que se obtienen al estudiar un fenómeno es una de las actividades fundamentales en toda investigación. En la simulación de redes, en particular, el formato de representación visual, a través de herramientas gráficas, ofrece una mejor interpretación del escenario simulado y permite comprender de manera más clara los parámetros analizados. La elección de las herramientas adecuadas para realizar esta tarea, así como el desarrollo de las competencias necesarias para manejarlas, son esenciales en el estudio y la enseñanza de conceptos relacionados a las redes de computadoras.

Este informe presenta de manera comparativa algunas herramientas que se utilizan en la representación gráfica de los resultados generados por el simulador NS-2. Se describen las ventajas y desventajas, alcances, limitaciones y requisitos para la utilización de cada una de ellas.

Palabras clave: Simulación de redes; Herramientas de simulación; Tráfico de red; Análisis gráfico.

\begin{abstract}
Data processing obtained from the study of a phenomenon is one of the fundamental activities in any research. In the network simulation, in particular, the format of visual representation, through graphic tools, offers a better interpretation of the simulated scenario and allows a clearer understanding of the parameters analyzed. Choosing the right tools to perform this task, as well as the development of the necessary skills to handle them, are essential in the study and teaching of concepts related to computer networking.

This paper presents in a comparative way some tools that are used in the NS-2 simulator graphic representation results. There's also an explanation of advantages and disadvantages, scope, limitations and requirements for the use of each one of them.
\end{abstract}

Key words: Network simulation; Simulation tools; Network traffic; Graphic analysis. 


\section{INTRODUCCIÓN}

Existen muchas áreas de investigación en las que se precisa contar con la representación gráfica de los datos. Para determinar qué herramienta de procesamiento gráfico utilizar en el estudio de redes de computadoras, será necesario conocer los aspectos más relevantes de cada una de las opciones disponibles. Un problema que habitualmente se presenta es que la cantidad de documentación disponible para cada una de ellas puede variar, desde ser virtualmente inexistente hasta ser extremadamente compleja. Por ello, se plantea la necesidad de una síntesis teórica que colabore en esta decisión.

Mediante este trabajo trataremos de determinar cuál de las herramientas es más apta para la realización de determinados tipos de análisis. Para ello, analizaremos las ventajas y desventajas de tres herramientas fiables, con una amplia variedad de funcionalidades y opciones de presentación. Estas características resultan fundamentales a la hora de generar gráficos que atraigan la atención del lector, presentando la información de forma sencilla, clara y precisa, ya que al simplificar la comparación de datos se facilita la comprensión del fenómeno estudiado.

Las herramientas seleccionadas para este análisis son gnuplot, Xgraph y TraceGraph. Cada una de ellas fue extensamente probada como herramienta gráfica de post-proceso de datos generados con el simulador NS-2.

El texto se encuentra organizado de la siguiente manera: En la Sección 2, se describe el marco histórico en el que surge el protocolo TCP, y cómo se originan los problemas que motivan este proyecto de investigación. Luego, en el marco conceptual, se definen los conceptos involucrados; y en el marco teórico, se presenta el fundamento teórico que motiva el análisis de las herramientas de graficación. La Sección 3, se enfoca en describir la metodología que se utiliza en esta investigación, para ello y con el fin de explicar el origen de los datos a graficar, se presenta una breve síntesis del enfoque de simulación y, en particular, de la herramienta NS-2 -el simulador de redes utilizado-, y luego, cada una de las herramientas de procesamiento gráfico seleccionadas. Mientras que, en la Sección 4, a partir del diseño y simulación de un modelo sencillo, se presentan los resultados de realizar los gráficos con las diferentes herramientas sobre el mismo conjunto de datos. En la Sección 5 se discuten estos resultados y se plantea un marco de evaluación para las herramientas, a partir del cual se analizan las ventajas y desventajas de cada una de ellas, y el ámbito donde cada una resulta más adecuada. Finalmente, en la Sección 6, se presentan las conclusiones que se pudieron obtener a partir del uso de estos sistemas de graficación de datos post-proceso y se plantean posibles trabajos a futuro.

\section{MARCO DE REFERENCIA}

\section{Marco histórico}

El protocolo TCP (Transmission Control Protocol) (Postel, 1981) es actualmente usado en gran parte de las comunicaciones de datos. Fue desarrollado en la década del '70 para implementarse en topologías cableadas, que eran las predominantes en la época. Sin embargo, con el surgimiento de las redes inalámbricas a finales de la década del ' 90 , el protocolo comenzó a evidenciar ciertas falencias. Esto se debe a que la pérdida de paquetes en topologías inalámbricas no siempre es causada por la congestión de la red como pasa frecuentemente en redes cableadas, para las que fue originalmente diseñado.

La pérdida de datos cuando hablamos de redes inalámbricas puede darse por una multiplicidad de factores, como desconexiones por pérdida de la señal, interferencias, ancho 
de banda limitado y variable, entre otros. Las posibles soluciones para distinguir el origen de las pérdidas se han implementado con la introducción de nuevos algoritmos en el protocolo, que se presentan como variantes de TCP.

Aunque cada una de las variantes intenta mitigar las deficiencias, con la resolución de algunos problemas se suelen introducir otros. Por lo que, a pesar de las mejoras significativas propuestas y llevadas a cabo a lo largo de los años, TCP continúa con ciertas deficiencias en las topologías inalámbricas que resultan en un deterioro en el rendimiento de la red. Por ello, es necesario continuar con investigaciones que puedan resolver el problema planteado y posibilitar un funcionamiento adecuado teniendo en cuenta el nuevo escenario.

En el ámbito de la investigación se utilizan simuladores de red para recrear los posibles escenarios, y luego, se utilizan los resultados obtenidos para diseñar gráficos que otorguen mayor claridad sobre el escenario simulado y permitan comprender de manera más tangible los conceptos involucrados.

\section{Marco conceptual}

La simulación de redes es, sin duda, una de las metodologías de evaluación predominantes en una gran variedad de áreas del conocimiento, y en particular en el área de redes informáticas. Es ampliamente utilizada para el desarrollo de nuevas arquitecturas de comunicación y protocolos de red (Weingärtner et al., 2009). En los ámbitos de investigación y educativos, donde la mayoría de las actividades se basan en entidades de simulación, y se trabaja con hipótesis y supuestos, las propuestas de trabajo están enunciadas en base a una topología determinada y un flujo de datos al cual está sometido el modelo que planteamos (Torres et al., 2015).

En el estudio de las redes, la simulación permite recrear el comportamiento de un sistema controlando los parámetros y configuraciones que influyen en una red, modelándola de manera arbitraria al especificar el comportamiento de cada uno de los nodos y los canales de comunicación (Weingärtner et al., 2009). Este enfoque da como resultado una extensa lista de datos sobre cada instante de la simulación, lo que resulta abrumador para llegar a conclusiones objetivas.

La representación gráfica es una forma de representar datos mediante recursos visuales que sirve al investigador para analizar el comportamiento de ciertos fenómenos (Allman \& Falk, 1999). Por ello, se recurre a la utilización de herramientas específicas para este fin, que permitan presentar los resultados combinando sencillez y precisión.

\section{Marco teórico}

La problemática de transmisión de datos en topologías mixtas que implican atravesar medios cableados e inalámbricos presenta un grado de complejidad específico que habilita su estudio en forma extensa. Para llevar adelante el análisis de las variantes del protocolo TCP, se recurre al ensayo de simulaciones con distintos tipos de herramientas de software, en particular el simulador NS-2 y, luego de procesar los resultados obtenidos, se recurre a una combinación de varias herramientas de procesamiento gráfico (Martínez B. \& Salvador, 2011).

Muchos trabajos de investigación han realizado estudios de desempeño de las variantes de protocolo TCP en distintos escenarios y para ello la representación de los resultados es una tarea básica y fundamental que permite elaborar los informes que se publican en estas investigaciones (González et al., s/f). 


\section{METODOLOGÍA}

\section{Herramientas de software y métodos utilizados}

Para el desarrollo de la investigación se utilizaron tres herramientas informáticas específicas para el procesamiento gráfico: gnuplot (v.5.0.7) (Williams et al., 2018), XGraph (v.12.1) (Pradeep Kumar, 2011) y Tracegraph (v.2.05) (Camacho L., s/f; Pradeep Kumar, 2012); el simulador de redes Network Simulator-2 (NS-2, v.2.35); y Network Animator (Nam, v.1.15), el visualizador gráfico que se instala junto al simulador. Asimismo, existen otras herramientas adicionales -como el software Matlab (Pandian, 2014)- que admiten los archivos de datos generados por NS-2 y permiten evaluarlos para su representación.

Además del ensayo con cada una de las herramientas, se recurrió a su documentación oficial, manuales e información disponible en los sitios web de aquellas que siguen siendo mantenidas por sus desarrolladores; en los casos en que esto no fue posible, se acudió a otras fuentes como blogs o trabajos informales realizados por la comunidad de usuarios.

La metodología en la investigación comienza con la formulación del problema. En esta fase de especificación, el investigador plantea el problema y presenta tanto los objetivos e hipótesis del estudio, como las características de la simulación a llevar a cabo.

Luego de reunir estos datos y definir el escenario a simular, se debe reflejar esto en un lenguaje que el simulador pueda comprender. Para ello se crean los scripts de la simulación en Otcl, una versión de Tcl2 orientada a objetos (Esterhuizen \& Aes, 2012). De esta manera se describe la topología de red, la pila de protocolos y parámetros específicos de cada uno de ellos, entre otras particularidades que pueden definirse.

Finalmente, se llevan a cabo las ejecuciones de simulación en NS-2, es decir, se ejecutan los scripts diseñados y luego se recopilan los resultados. No obstante, los resultados obtenidos del simulador no se encuentran en un formato fácilmente legible para humanos. Y aunque estas trazas pueden ser leídas y entendidas línea a línea por el investigador, resultaría difícil llegar a resultados objetivos a partir de cada observación aislada.

Por ello es importante advertir que cuando las actividades científicas producen una gran cantidad de datos, también requieren de cierto grado de procesamiento previo a su utilización. Y así como existe una variedad de datos científicos, también hay gran variedad de herramientas que permiten su visualización, análisis, procesamiento y edición (Pölcher Núñez et al., 2012).

En este trabajo se realizará un análisis que describirá de manera comparativa las principales herramientas de graficación que se utilizan en el ámbito de investigación en redes informáticas, y como complemento al simulador NS-2, para producir los gráficos que permitirán a los investigadores facilitar el análisis y documentar las conclusiones.

Así, tomando como referencia el marco de trabajo utilizado en (Calle et al., 2018; Velásquez \& Gamess, 2009), se define en primera instancia, un conjunto estandarizado de parámetros y criterios que se utilizarán para el análisis y comparación de las tres herramientas de graficación seleccionadas.

\section{Parámetros a analizar para elegir una herramienta de graficación}

A continuación se presentan parámetros que resultan útiles para el análisis comparativo de las tres herramientas de graficación seleccionadas sobre una base homogénea, lo que facilita notablemente el proceso de selección. Además, estos parámetros podrían ser utilizados para ampliar, de ser necesario, la lista de herramientas. En la Sección 4, la Tabla 1 corresponde a una matriz donde para cada una de las diferentes herramientas de graficación analizadas, se presenta el valor de cada uno de los parámetros aquí descritos. La tabla 1, por lo tanto, se constituye en una herramienta rápida de comparación y selección (Calle et al., 2018). 


\section{Versión actual y última actualización}

La fecha de la última actualización es importante, ya que muestra qué tan activo es el proyecto, mientras que el número de versión actual indica sobre qué versión de las herramientas se hizo el análisis en este trabajo.

\section{Plataformas soportadas (GNU/Linux, MS Windows, Otros)}

Es indispensable saber de antemano si las herramientas son compatibles con el sistema operativo que el investigador o estudiante utiliza habitualmente. Si bien éste no es un impedimento para utilizarlas (se puede recurrir a máquinas virtuales, por ejemplo), el soporte de la plataforma a la que está familiarizado el usuario será una característica básica a tener en cuenta.

\section{Licencia y derechos de autor}

La licencia de software, así como los derechos de autor son características que se deben conocer siempre al elegir una herramienta. Es común que el investigador, debido a las particularidades de sus datos o de sus objetivos de investigación, necesite desarrollar algoritmos de análisis y/o de procesamiento propios. Esta posibilidad de desarrollar algoritmos propios no siempre está incorporada en el software privativo [13].

\section{Presentación (Interfaz gráfica (GUI), Línea de comandos (CLI))}

En cuanto a la presentación de la interfaz, no se puede aducir que la interfaz por línea de comandos sea superior a la interfaz gráfica o viceversa, no obstante la elección de la misma dependerá de la experiencia y preferencia de cada usuario, así como de cada situación de uso. Por ello, al elegir la herramienta adecuada, es un aspecto a tener en cuenta que la misma trabaje con el tipo de interfaz con la que el usuario se sienta más cómodo.

\section{Formato de entrada (Requiere procesamiento previo, No requiere procesamiento previo)}

El formato de los datos de entrada es una característica que interesa evaluar, dado que el procesamiento previo de los datos es una tarea que lleva cierto tiempo y esfuerzo por parte del investigador. La admisión de archivos comprimidos por parte de la herramienta, otorga además un ahorro en el espacio de almacenamiento.

\section{Exclusivo para procesar salidas de NS-2}

Aunque en el presente trabajo las herramientas analizadas se utilizaron en conjunto con el simulador NS-2, se puede apreciar que algunas de ellas son sólo exclusivas para este uso. Si bien no es una característica esencial que las herramientas puedan ser destinadas al uso en combinación con otro software, es una peculiaridad que podría ser de interés en ámbitos donde se requiera graficar también otro tipo de datos.

\section{Uso (Interactivo, Mediante scripts (automatización))}

El uso interactivo de la herramienta es útil en la realización de gráficos individuales o únicos. Pero es de vital importancia al seleccionar la herramienta de graficación -particularmente en el área de investigación- evaluar si la misma admite también el uso mediante scripts. Esto permite la automatización en la generación de gráficos a partir de múltiples datos de entrada, y resulta en un consecuente ahorro de tiempo y esfuerzo.

Dimensión de las gráficas (Bidimensional, Tridimensional), Gráficos de múltiples valores superpuestos y Grado de personalización de las gráficas.

El usuario deberá tomar decisiones acerca de sus requerimientos respecto a las gráficas resultantes. En consecuencia, es su misión determinar si precisa que las gráficas sean 
bidimensionales o tridimensionales. Además, que las gráficas puedan expresar múltiples valores superpuestos potencia la explicación del usuario, resultando claramente útil para la comparación directa de diferentes valores, por lo que es importante considerar este ítem. Igualmente, el grado de personalización en relación a los estilos de líneas, colores, títulos y ubicación de cada elemento, entre otros componentes de las gráficas, es diferente en cada una de las herramientas. Por ello es necesario que se valore también este aspecto.

\section{Impresión y Formato de salida}

De acuerdo a la finalidad de los gráficos, es de suma importancia estudiar los formatos de salida que ofrece cada herramienta, así como también la posibilidad de imprimir directamente los gráficos desde la misma.

\section{Documentación y ayuda disponible (Inexistente, Escasa, Precisa y completa)}

La cantidad de documentación, y exactitud de la misma, es un aspecto valioso a la hora de utilizar una herramienta nueva. Además, la disponibilidad de una página web oficial, foros de usuarios, manuales de uso y recopilación de FAQ (preguntas frecuentes) resultan de suma utilidad para resolver dudas e inconvenientes en el uso de cualquier aplicación.

\section{Curva de aprendizaje requerida (Fácil uso, Requiere entrenamiento)}

Aprender su utilización y resolver las dificultades que se encuentren de manera adecuada, son también objetivos relacionados con la curva de aprendizaje, y pueden determinar que el estudiante o investigador se anime a conocer cada detalle de su funcionamiento y hacerse hábil en el uso de la herramienta, o bien, abandone con frustración su uso.

\section{RESULTADOS}

El simulador NS-2 puede generar dos tipos de resultados, ficheros de salida con extensión .nam o .tr.

El primero es un fichero de animación que puede ser ejecutado mediante Nam, una herramienta, comúnmente instalada junto al simulador, que facilita la interpretación de los resultados de la simulación en NS-2, permitiendo visualizarlos dinámicamente (Pellicer C., 2010). De esta manera, posibilita la creación de animaciones en las que se visualiza la topología, distinguiendo los tipos de paquetes, nodos y enlaces, mediante la asignación de colores, formas y etiquetas diferentes. A medida que avanza, se puede hacer el análisis de paquetes, ya que al pulsar cada uno de ellos, se obtiene el tipo, tamaño y número de secuencia. Nam ofrece, además, opciones para guardar estas animaciones en formato de video MPEG o imágenes GIF. En la figura 1 se puede observar la interfaz y la captura de una animación en curso, correspondiente al mismo modelo que utilizaremos posteriormente para analizar las herramientas específicas de procesamiento gráfico. 


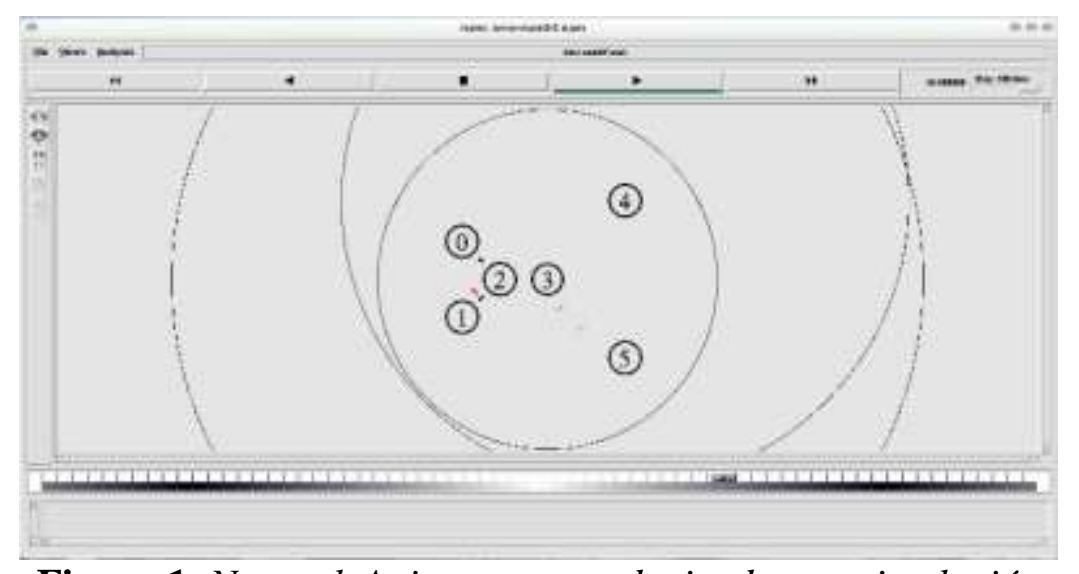

Figura 1. Network Animator reproduciendo una simulación

Aunque esta herramienta permite ejecutar solo un ensayo por vez, resulta un complemento adecuado para NS-2, ya que posibilita una rápida validación de la simulación realizada a partir de un análisis visual del escenario que debe coincidir con el modelo del sistema que se pretendía configurar. A pesar de las ventajas presentadas que impulsan su uso, en el ámbito de la investigación resulta insuficiente y requiere complementarse con otras herramientas de representación gráfica que presentaremos en las siguientes secciones.

El segundo tipo de resultado que brinda NS-2 es la generación de archivos de traza. Dado que el objetivo de la simulación no es conocer el modelo en sí, sino el comportamiento que este tiene ante situaciones particulares, esta es la salida más informativa, que además permite múltiples ejecuciones a la vez, lo que asegura su escalabilidad.

En la Figura 2 se muestra un esquema del proceso general de simulación que finaliza con los dos tipos de salida descriptos, el archivo para ejecutar la animación, o bien, los archivos de traza para el procesamiento posterior y la ejecución de múltiples tipos de gráficos y análisis.

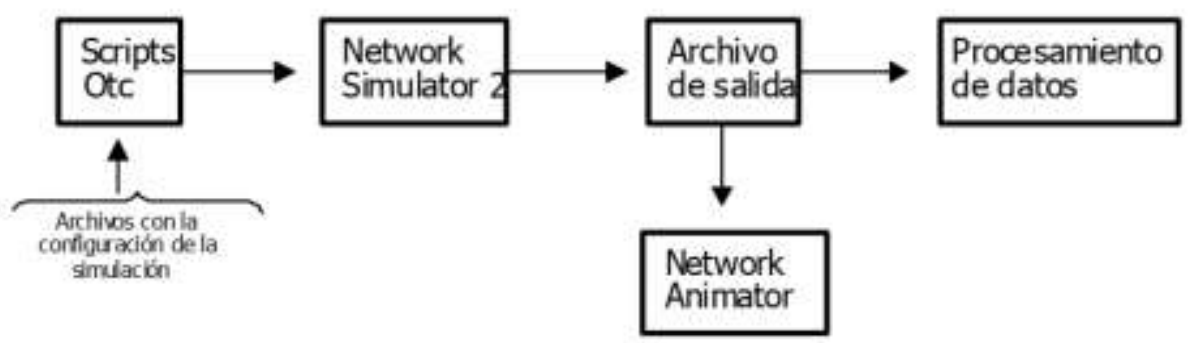

Figura 2. Estructura del proceso de simulación

Los archivos de traza muestran información relevante de la simulación realizada. A partir de ellos, se pueden obtener algunos parámetros de rendimiento de la red, que permiten estudiar el comportamiento de distintos fenómenos como congestión (Esterhuizen \& Aes, 2012; Jacobson, 1988), colisiones y competencia por recursos (González et al., 2019), entre otros. Es tanta la información que puede contener una traza, que habitualmente las herramientas para graficar requieren de un proceso de filtrado previo que prepare estos archivos de acuerdo a los gráficos que se desee obtener.

Para realizar el tratamiento de la información, tomaremos una topología híbrida (Pandey \& Tyagi, 2013; Pentikousis, 2000) como la de la figura 3. En base a ella se realizó la simulación y se generó la base de datos que fue posteriormente representada con las tres herramientas gráficas que se analizan. 


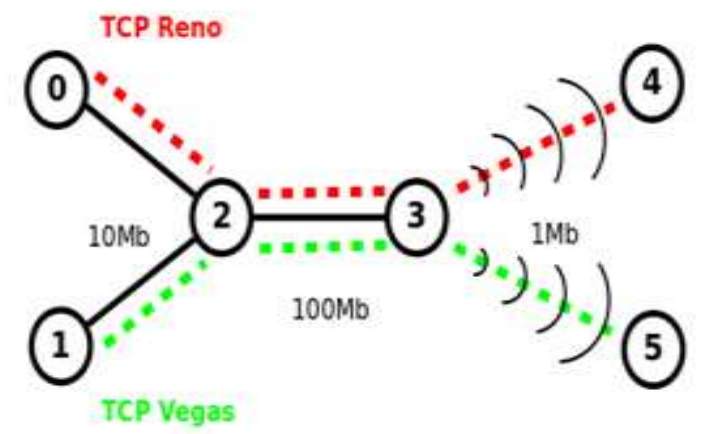

Figura 3. Modelo de topología híbrida a simular en NS-2.

Las herramientas utilizadas para generar gráficos a partir de los archivos de traza obtenidos, directa e indirectamente de esta simulación fueron gnuplot, XGraph y TraceGraph.

gnuplot (con la $\mathrm{g}$ en minúsculas) es una herramienta de línea de comando interactiva para generar gráficos como el observado en la figura 4. Puede generar funciones y puntos en dos o tres dimensiones, en diferentes formatos. Fue diseñada principalmente para la muestra visual de datos científicos, las imágenes son de excelente calidad y pueden ser incluidas en publicaciones sin mayores modificaciones (Pölcher N. et al., 2012). Está disponible para GNU/Linux, MS Windows, OSX y derivados de BSD, entre otros sistemas operativos. Adicionalmente, el código fuente aún soporta sistemas más antiguos como VMS, Ultrix, OS/2 y MS-DOS. Aunque está protegido por derechos de autor, el código fuente es de distribución libre.

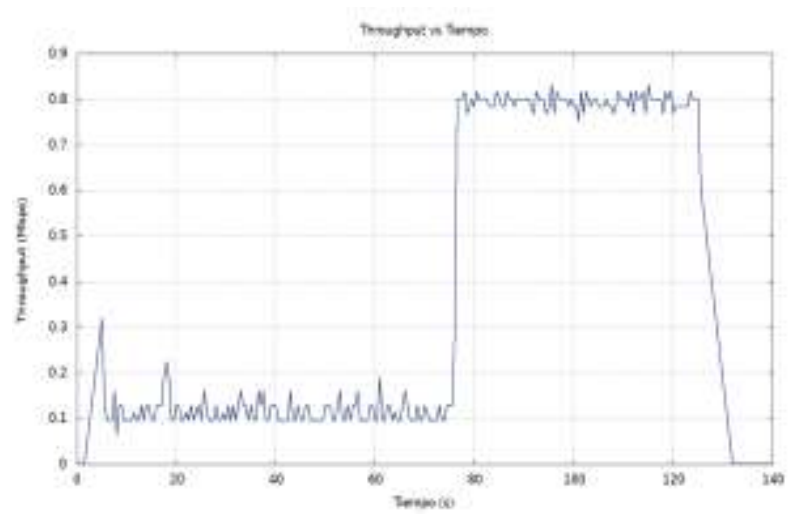

Figura 4. Gráfico de throughput obtenido mediante un script en gnuplot.

Una de las características distintivas de esta aplicación es que, además de poder ser utilizada mediante línea de comandos, se pueden encontrar diferentes frentes gráficos que facilitarán la tarea a los usuarios menos experimentados. Uno de ellos es PlotDrop, que ofrece una interfaz sencilla con las funcionalidades más requeridas a la hora de realizar gráficos y proporciona información de la evolución de una variable como se observa en la figura 5. 


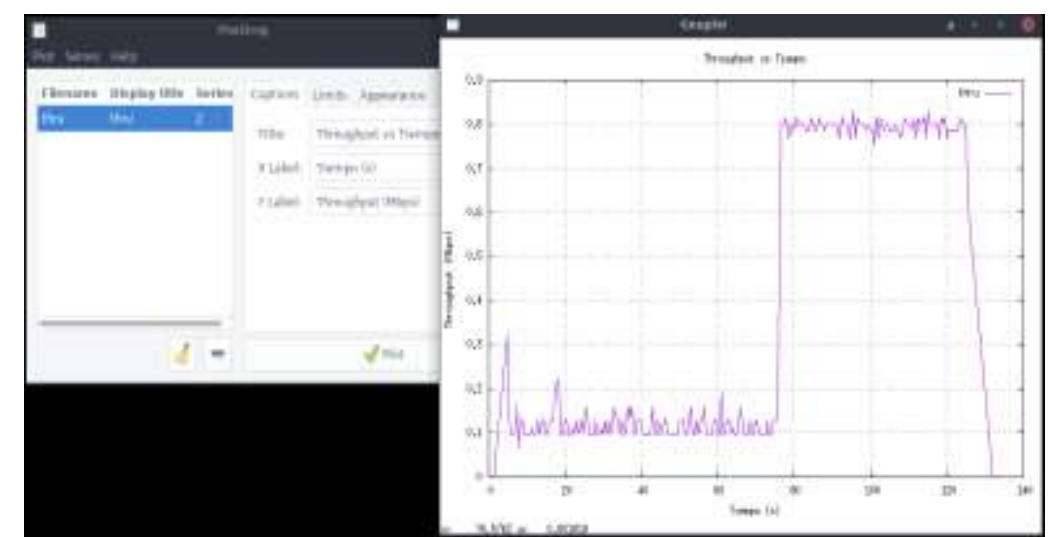

Figura 5. Gráfico de throughput obtenido mediante la interfaz gráfica PlotDrop.

XGraph es una herramienta sencilla para la representación gráfica de un conjunto de datos. Es un graficador bidimensional de propósito general, con botones interactivos que permite panoramizar, ampliar, imprimir y seleccionar opciones de visualización. Puede manejar un tamaño ilimitado de conjuntos de datos, así como cantidad de archivos; permite, además, renombrar los títulos, numerar y etiquetar los ejes o leyendas, y grabar los gráficos como ficheros PostScript (Pellicer Corbacho, 2010). Está disponible para GNU/Linux, MS Windows, Apple Mac OS-X, FreeBSD y Sun Solaris. Esta herramienta fue presentada en 1985 y continúa siendo mejorada, utilizada y respaldada por varias organizaciones y proyectos.

Es destacable que XGraph permita trabajar de dos maneras, interactiva o no interactiva, según se utiliza su interfaz gráfica o mediante línea de comandos para procesar archivos por lotes. Además, archivos comprimidos como entrada, reduciendo considerablemente el espacio de almacenamiento necesario. En la figura 6, se puede observar la forma en que esta aplicación representa la información mediante un gráfico.

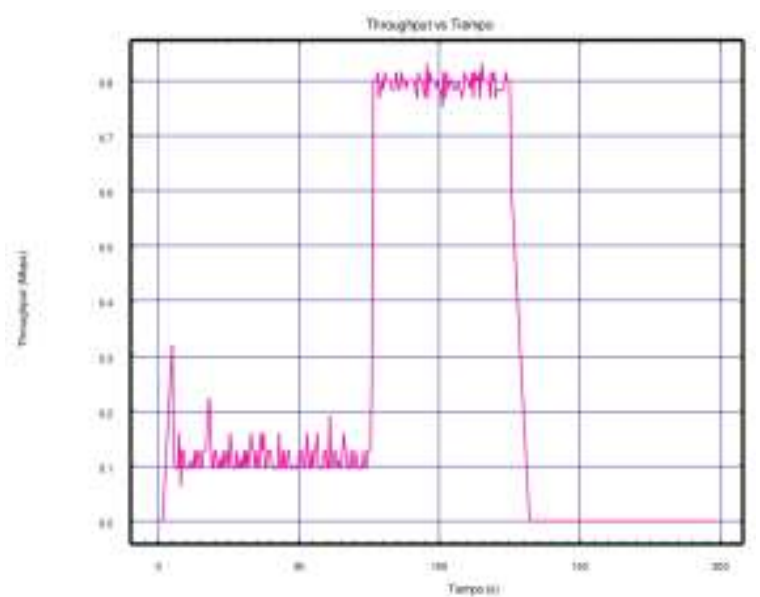

Figura 6. Gráfico de throughput generado mediante XGraph.

TraceGraph es la tercera de las herramientas seleccionadas para este análisis. Se encuentra disponible tanto para GNU/Linux como MS Windows. Dado que esta aplicación utiliza las librerías de Matlab para su funcionamiento, éstas también deben ser instaladas. Ofrece una amplia variedad de opciones para graficar: doce tipos de gráficos tridimencionales y cientos de tipos de gráficos bidimensionales. Además, permite analizar distintos formatos de traza obtenidos mediante el simulador NS-2, instalando una extensión denominada Trace Converter para graficar la información contenida en el archivo .tr sin necesidad de aplicar ningún otro proceso a los datos de entrada. 


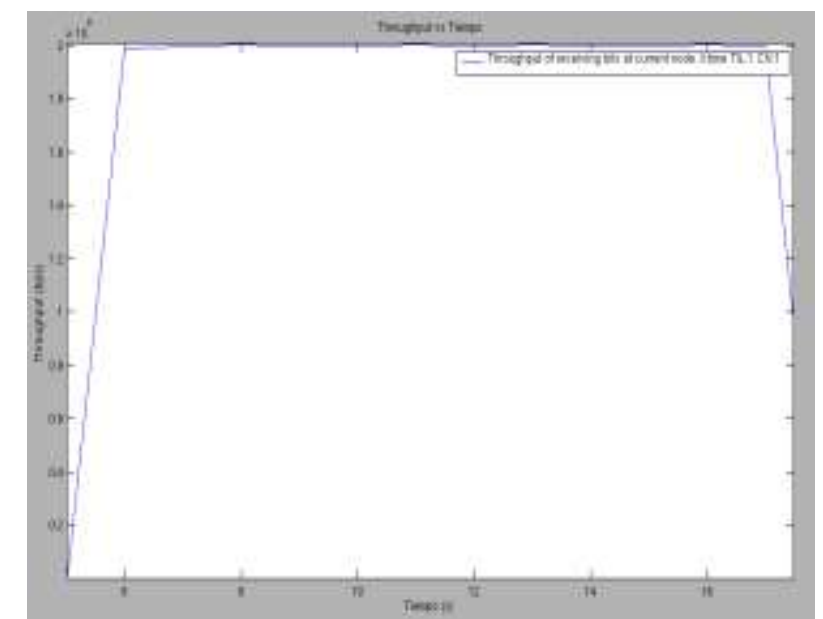

Figura 7. Gráfico de throughput generado mediante TraceGraph.

Por razones que se desarrollarán en la discusión, el gráfico presentado para TraceGraph, que se puede observar en la figura 7 , se corresponde a un modelo diferente, con las mismas especificaciones pero con dos nodos cableados y un solo enlace de $2 \mathrm{Mb}$.

\section{DISCUSIÓN}

Debido a la falta de documentación encontrada para el uso de TraceGraph, no se pudo resolver la realización de gráficos a partir de trazas resultantes de un modelo híbrido cableado-inalámbrico como el caso de estudio planteaba. Por ello, se utilizó un modelo cableado sencillo para mostrar el resultado que se obtiene con la herramienta.

Como era de esperar, los datos representados no cambian de una herramienta a otra. Las diferencias visuales se manifiestan en el estilo y grosor de la línea, color, escalas, fuentes, etc., pero los aspectos que varían de forma más significativa entre una y otra, se refieren al trabajo involucrado: cuánto se tardó en generar cada gráfico, desde el procesamiento de los archivos de traza (si la herramienta lo requería) hasta la obtención del gráfico deseado; cuánto conocimiento técnico en el área y acerca de la herramienta es requerido para poder graficar los datos; cuántos inconvenientes debidos a fallas en el software o en la documentación aparecieron en el proceso.

Los distintos grados de esfuerzo involucrado hacen que las herramientas no sean adecuadas para cualquier situación. Estas son algunas de las observaciones que surgen a partir del uso y estudio de la documentación de las herramientas:

- Un caso recurrente en el estudio de redes de computadoras es la automatización de simulaciones y el uso de scripts para generar gráficos de los datos a medida que se van obteniendo. Por lo que las herramientas gnuplot y XGraph se destacan por encima de TraceGraph a la hora de realizar procesamiento por lotes.

- No necesitar procesamiento de los archivos de traza hace que TraceGraph sea más adecuada para el análisis inmediato de simulaciones individuales.

- La documentación de gnuplot es más precisa y completa que la de las demás, por lo que resulta la herramienta más confiable en un entorno de producción.

- Para reducir la cantidad de almacenamiento requerido por los datos a graficar, es conveniente utilizar XGraph, dado que admite entradas en formato comprimido.

- Si la necesidad de personalización y detalles en los gráficos es alta, gnuplot es ideal, aunque XGraph también cumple con los requisitos. En este caso, TraceGraph resulta inadecuada. 
- Si se quiere introducir en el uso de estas herramientas y se necesita recurrir a la documentación, gnuplot y XGraph, en menor medida, disponen de muchos recursos a los que se puede acudir, como foros, tutoriales, guía de preguntas frecuentes y manuales de uso, entre otros. TraceGraph es difícil de utilizar debido a su falta de documentación.

A continuación se realiza un compendio de las diferencias más importantes detectadas en el estudio de las herramientas, considerándose los parámetros comparación definidos en la Sección 3, como última actualización, plataformas soportadas, tipo de licencia, tipo de interfaz - gráfica, por línea de comandos, o ambas-, el formato de entrada de los datos y los posibles formatos de salida para los gráficos resultantes, entre otros aspectos. Se realiza la evaluación de cada uno de ellos, en las tres herramientas de graficación seleccionadas, con el fin de establecer un cuadro comparativo (Tabla 1) que le permita al usuario visualizar de una manera clara las ventajas y desventajas de elegir una herramienta u otra (Calle et al., 2018).

Tabla 1. Parametrización de las herramientas de graficación evaluadas.

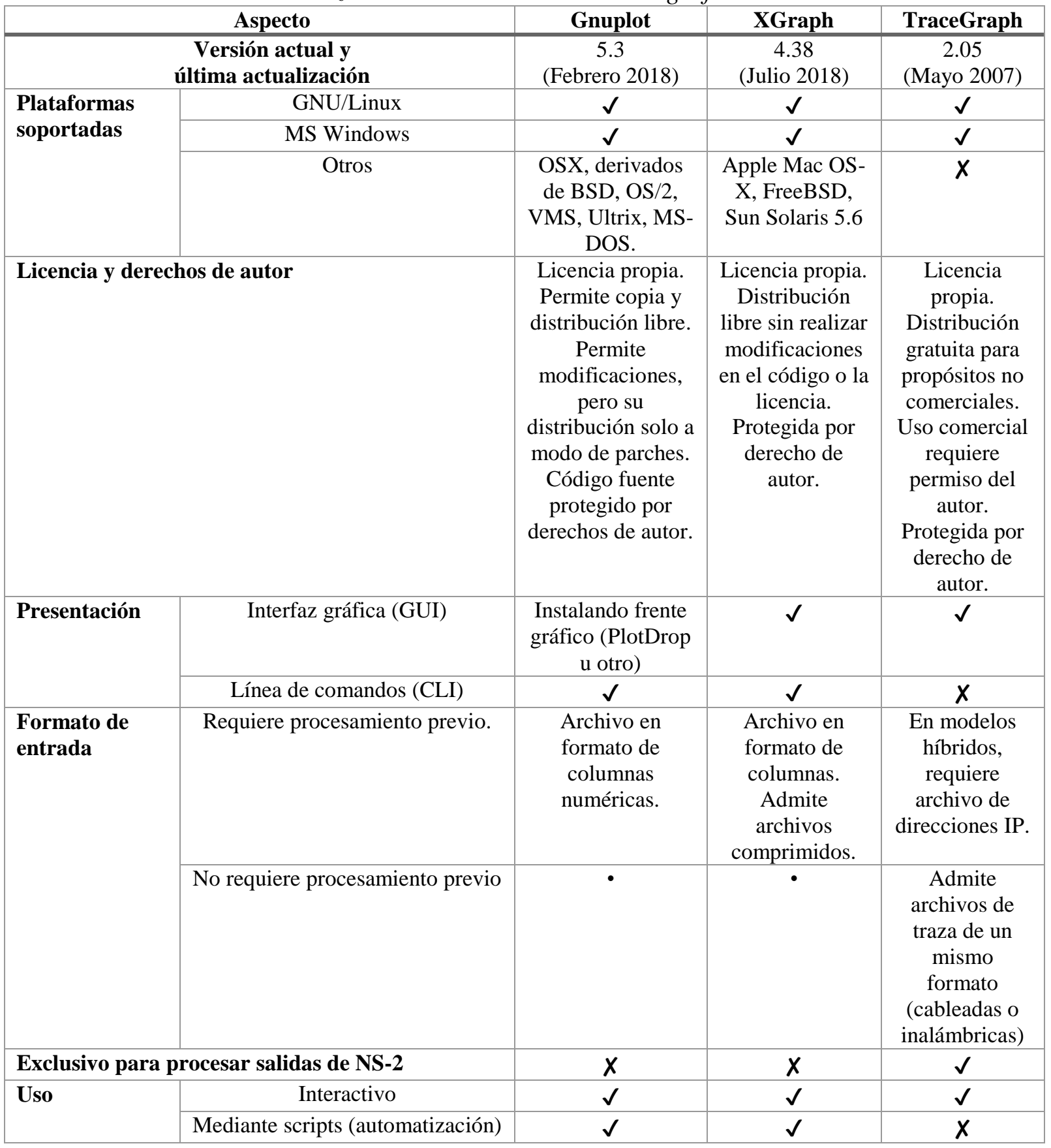




\begin{tabular}{|c|c|c|c|c|}
\hline \multirow{2}{*}{$\begin{array}{l}\text { Dimensión de } \\
\text { las gráficas }\end{array}$} & Bidimensional & $\checkmark$ & $\checkmark$ & $\checkmark$ \\
\hline & Tridimensional & $\checkmark$ & $x$ & $\checkmark$ \\
\hline \multicolumn{2}{|c|}{ Gráficos de múltiples valores superpuestos. } & $\checkmark$ & $\checkmark$ & $\checkmark$ \\
\hline \multicolumn{2}{|c|}{ Grado de personalización de las gráficas. } & Alto. & Aceptable. & Bajo. \\
\hline \multicolumn{2}{|l|}{ Impresión } & $\checkmark$ & $\checkmark$ & $x$ \\
\hline \multicolumn{2}{|c|}{ Formato de salida } & $\begin{array}{l}\text { PNG, EPS, SVG, } \\
\text { JPEG, GIF, } \\
\text { POST, WXT, } \\
\text { WINx11 }\end{array}$ & $\begin{array}{l}\text { PostScript, } \\
\text { PDF, PPTX, } \\
\text { ODP, PPM, } \\
\text { JPG, PNG, GIF. }\end{array}$ & $\begin{array}{l}\text { Archivos de } \\
\text { texto, gráficos } \\
\text { JPG y TIFF }\end{array}$ \\
\hline \multirow{3}{*}{$\begin{array}{l}\text { Documentación } \\
\text { y ayuda } \\
\text { disponible }\end{array}$} & Inexistente & • & $\cdot$ & $\begin{array}{l}\text { Sólo blogs } \\
\text { informales. }\end{array}$ \\
\hline & Escasa & $\cdot$ & $\begin{array}{l}\text { No se encuentra } \\
\text { actualizada. }\end{array}$ & $\cdot$ \\
\hline & Precisa y completa & $\checkmark$ & $\cdot$ & $\bullet$ \\
\hline \multirow{2}{*}{$\begin{array}{l}\text { Curva de } \\
\text { aprendizaje } \\
\text { requerida }\end{array}$} & Fácil uso & $x$ & $x$ & $x$ \\
\hline & Requiere entrenamiento & $\checkmark$ & $\checkmark$ & $\checkmark$ \\
\hline
\end{tabular}

\section{CONCLUSIONES}

La representación visual de los datos obtenidos en una investigación resulta fundamental para lograr una comprensión íntegra del fenómeno estudiado.

Seleccionar las herramientas adecuadas que permita graficar los resultados de una simulación generada con NS-2 y utilizarlas apropiadamente, es una tarea necesaria y resulta de gran ayuda al momento de realizar un análisis.

Mediante la experimentación con estas herramientas, se advirtió que no es posible establecer un modelo general para procesar y analizar los datos de una simulación, ni una única herramienta suficiente para satisfacer todas las necesidades de la investigación puesto que depende del objetivo del estudio, de las necesidades a cubrir, de las fuentes de información con que se cuenta, de los recursos y de las prioridades. De manera que la elección y el dominio de una herramienta adecuada para la representación gráfica no son de carácter excluyente, sino que se adapta a las necesidades planteadas por la tarea a realizar, se plantea la necesidad de emplear herramientas combinadas.

Para ayudar en la tarea del investigador, instructor o estudiante, la herramienta ideal debería mantener el foco en los datos, y no en detalles técnicos de su uso. Y por ello, es esencial que las herramientas que se elijan sean actuales y de proyectos activos, en lo posible, dado que esto reduce la tarea en la búsqueda de información para implementar las funcionalidades de las herramientas en cuestión y evita posibles errores de compatibilidad.

El conocimiento en el área y la aplicación de distintas herramientas permite a estudiantes e investigadores seleccionar aquellas que resulten óptimas para cada situación. En el ámbito de la investigación, resultaron de suma utilidad las herramientas que permiten la creación de scripts para automatizar ciertas tareas, y aunque esto exige ciertos conocimientos y/o habilidades de programación, se ve compensado en el ahorro de tiempo y esfuerzo. También, cabe agregar que una buena parte de la experiencia y el conocimiento adquirido con el uso de una de herramientas, actúa de manera sinérgica, permitiendo y facilitando el acceso a las otras, o incluso a otras de naturaleza similar.

Aunque en la actualidad las herramientas presentadas son las más destacadas en el área de graficación en simulaciones de redes, queda la posibilidad de expandir la lista de herramientas y modificarla en trabajos futuros, para adaptarse a las nuevas necesidades en el ámbito de 
investigación de redes de computadoras. En este sentido, el marco de evaluación aquí planteado resultaría una herramienta de comparación y selección válida.

\section{RECOMENDACIONES}

En el transcurso de una investigación suele surgir la necesidad de utilizar una representación visual del problema. Esto permite decidir y comunicar con mayor confianza qué se va a hacer, evaluar la coherencia de los resultados obtenidos o comunicar las conclusiones consecuentes. Como se ha mencionado, las herramientas de representación gráfica pueden tener usos variados según la etapa de investigación en la que se encuentre.

Se pueden utilizar como guía los consejos dados en la discusión para decidir en qué caso sería más adecuado la elección de una herramienta u otra. Sin embargo, no se recomienda familiarizarse con el uso de una sola de ellas, dado que las elecciones en este caso no suelen ser excluyentes, y en etapas diferentes podría ser indicado el uso de otra herramienta.

\section{AGRADECIMIENTOS}

Agradecemos a la Unidad Académica Río Gallegos, de la Universidad Nacional de la Patagonia Austral, por el financiamiento de este proyecto de investigación. Al Ing. Diego Rodríguez Herlein, la Lic. Claudia González, y el Sr. Franco Trinidad por la colaboración.

\section{REFERENCIAS}

ALLMAN, M., y FALK, A. (1999, Oct.). On the Effective Evaluation of TCP. SIGCOMM Comput. Commun. Rev., 29(5), 59-70. https://doi.org/10.1145/505696.505703

CALlE, M. A., TOVAR, J. D., CASTAÑO-PINO, Y. J., y CUÉllAR, J. C. (2018, Dic.). Comparación de parámetros para una selección apropiada de herramientas de simulación de redes. Información Tecnológica, 29(6), 253-266. https://doi.org/10.4067/s0718$\underline{07642018000600253}$

CAMACHO L., F. (s/f). Tracegraph y sus numerosas utilidades. Recuperado de Universidad Tecnológica Metropolitana (UTEM) website: https://bit.ly/2NYbQNm

ESTERHUIZEN, A., y AES, K. (2012). TCP Congestion Control Comparison. Southern Africa Telecommunication Networks and Applications Conference (SATNAC). 2-5 de septiembre de 2012, George. Recuperado de https://bit.ly/2SlFDyf

GONZÁleZ, C. N., RODRÍGUEZ H., D. R., TALAY, C. A., TRINIDAD, F. A., y ALMADA, M. L. (2019). Análisis de rendimiento y equidad en TCP. XXI Workshop de Investigadores en Ciencias de la Computación (WICC 2019), 106-109, 25-26 de abril de 2019. San Juan: Editorial UNSJ. https://doi.org/10.4067/s1726-569x2019000100137

GONZÁLEZ, C. N., TRINIDAD, F. A., ALMADA, M. L., TALAY, C. A., RODRÍGUEZ H., D. R., y MARRONE, L. A. (aceptado-en prensa). Herramientas para el procesamiento gráfico de datos en la simulación de redes. Revista Iberoamericana de Tecnología en Educación y Educación en Tecnología (TE\&ET). https://doi.org/10.24215/18509959.21.e07

JACOBSON, V. (1988). Congestion avoidance and control. ACM SIGCOMM Computer Communication Review, 18(4), 314-329. https://doi.org/10.1145/52325.52356 
MARTÍNEZ B., O., y SALVADOR, C. P. (2011). Introducción a la programación de protocolos de comunicaciones con Network Simulator 2. San Vicente: Editorial Club Universitario.

PANDEY, S., y TYAGI, V. (2013, Jun.). Performance Analysis of Wired and Wireless Network using NS2 Simulator. International Journal of Computer Applications (IJCA), 72(21), 38-44. https://doi.org/10.5120/12669-9404

PANDIAN, C. (2014). How to do ns2 trace analysis under Matlab using Tracegraph Tool. Recuperado el 17 de junio de 2019, de https://bit.ly/2IOcas9

PELLICER C., R. (2010). Estudio comparativo de la implementación de los protocolos RTP/RTCP en los simuladores Opnet Modeler y Network Simulator 2. Universidad Politécnica de Valencia, Escuela Politécnica Superior de Gandia. Tesis de Ing. de Sist. de Telecomunicación. Recuperado de https://bit.ly/2SIFScD. https://doi.org/10.4995/thesis/10251/5767

PENTIKOUSIS, K. (2000, Oct.). TCP in wired-cum-wireless environments. IEEE Communications Surveys \& Tutorials, 3(4), 2-14. https://doi.org/10.1109/comst.2000.5340805

PÖLCHER N., P., SALA, H., y MATKO, C. (2012). Herramientas de Software Libre para el análisis de datos científicos. Breve sinopsis y ejemplos aplicados al estudio del Sector Antártico Argentino. $3^{\circ}$ Conferencia Internacional de Software Libre (CISL 2012), 1-15, 15-17 de octubre de 2012, CABA. https://doi.org/10.5944/ried.1.10.1016

POSTEL, J. (1981). RFC 793: Transmission control protocol. DARPA.

PRADEEP KUMAR, T. (2011). Xgraph. Recuperado el 17 de junio de 2019, de https://bit.ly/2SoVV9C

PRADEEP KUMAR, T. (2012). Tracegraph - a graphing software to plot the trace files from NS2. Recuperado el 17 de junio de 2019, de https://bit.ly/2ZyqCLx

TORRES, J. A., FIGUEROA, D. A., y DÍAZ, J. (2015). Herramientas de software de simulación para redes de Comunicaciones. Recuperado de https://bit.ly/2SoxF7j

VELÁSQUEZ, K., y GAMESS, E. (2009). Análisis comparativo de herramientas de evaluación de desempeño en redes de computadores. En E. de Computación (Ed.), Lecturas en Ciencias de la Computación (1a ed.). Caracas: Universidad Central de Venezuela. https://doi.org/10.14483/2248762x.8503

WEINGÄRTNER, E., VOM LEHN, H., y WEHRLE, K. (2009). A performance comparison of recent network simulators. 2009 IEEE International Conference on Communications, 1-5, 14-18 de junio de 2009, Dresden. https://doi.org/10.1109/icc.2009.5198657

WILLIAMS, T., KELLEY, C., BERSCH, C., BRÖKER, H.-B., CAMPBELL, J., CUNNINGHAM, R., ... ZELLNER, J. (2018). gnuplot 5.2 An Interactive Plotting Program. Recuperado de https://bit.ly/2XUF8w7 